\title{
Performance Analysis of CDMA WLL Systems with Imperfect Power Control and Imperfect Sectorization
}

\author{
Sami A. El-Dolil \\ Department of Electronic and Electrical Communication Engineering, Faculty of Electronic Engineering, \\ Menoufiya University, Menouf 32952, Egypt \\ Correspondence should be addressed to Sami A. El-Dolil, msel_dolil@yahoo.com
}

Received 20 September 2007; Accepted 18 July 2008

Recommended by Mohsen Guizani

Wireless local loop (WLL) provides reliable, flexible, and economical access to the local telephone service using radio technology in the place of traditional wireline. In this paper, an analytical model is derived to evaluate the effect of both imperfect power control and imperfect sectorization on the performance of code division multiple access (CDMA) WLL systems. The results show that the capacity degradation, due to imperfect power control, is about $25.8 \%$ and $11.5 \%$ for single cell and multiple cell systems, respectively. Increasing the overlapping angle from $0^{\circ}$ to $5^{\circ}$ causes the capacity gain to decrease from 6 to 5.53 , while the corresponding sectorization efficiency drops from $100 \%$ to $92.3 \%$.

Copyright (C 2008 Sami A. El-Dolil. This is an open access article distributed under the Creative Commons Attribution License, which permits unrestricted use, distribution, and reproduction in any medium, provided the original work is properly cited.

\section{Introduction}

Wireless local loop (WLL) is a system that connects subscribers to the public switched telephone network (PSTN) using radio signals as a substitute for wireline for all or part of the connection between the subscribers and the switch. It is believed to be a fast and cost-effective mean to provide local phone service in rural areas and third world countries [1]. Since WLL is a fixed radio communication system; narrowbeam antennas can be employed at both the base station (BS) and subscriber's side so that the propagation between BS and subscriber's equipment is very close to free space propagation. This gives many inherent advantages to the WLL system over the traditional cellular systems, such as wider coverage area, reduced interference, higher capacity, no fast fading, and no handoff $[2,3]$.

CDMA technology has the potential to provide a significant improvement in the capacity of cellular radio systems compared with FDMA and TDMA systems [4]. However, this improvement is dependent upon the effectiveness of the power control system, especially on the reverse link. In the absence of power control, a BS would receive a much stronger signal from a subscriber unit that is geographically close to it than from a subscriber unit that is farther away. This is the so-called near-far problem $[5,6]$.
This paper presents a theoretical model to evaluate the reverse-link capacity of CDMA WLL systems in terms of outage probability, taking into account the power control error.

Sectorization in cellular CDMA systems increases the capacity in proportion to the number of sectors per cell. In practice, the antenna patterns do not fit the sector area perfectly, and there are overlapping between sectors, which causes additional interference on both the reverse and forward link [7]. The imperfect sectorization effect on the performance of CDMA WLL systems is also considered.

\section{Effect of Imperfect Power Control in Single Cell CDMA WLL Systems}

Consider a CDMA WLL single cell system consists of $N$ subscriber units transmitting to a $\mathrm{BS}$ receiver on the reverselink. A simplified CDMA transmitter is shown in Figure 1.

The signal transmitted from the $i$ th user to its BS is given by [5]

$$
S_{i}(t)=\sqrt{2 A_{i}} b_{i}(t) c_{i}(t) \cos \left(w_{1} t+\theta_{i}\right),
$$

where $A_{i}$ is the transmitted power of the $i$ th user, $b_{i}(t)$ is the data sequence of the $i$ th user, where each bit has an amplitude 


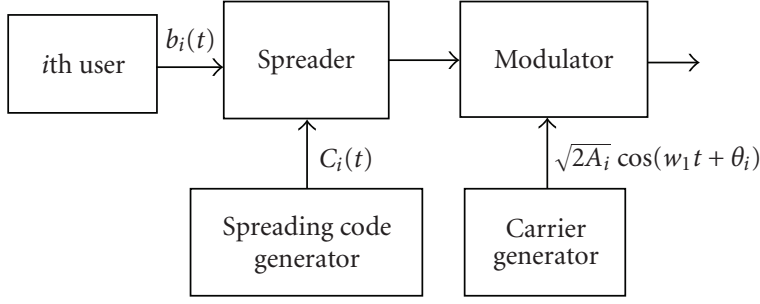

FIgURE 1: A simplified CDMA transmitter diagram.

of \pm 1 and a duration of $T_{b}, c_{i}(t)$ is the spreading code sequence of the $i$ th user and each of the $M$ chips per code has a duration $T_{c}, w_{i}$ is the reverse link-carrier frequency, and $\theta_{i}$ is the random phase of the $i$ th user carrier.

The received signal at the $\mathrm{BS}$ receiver $R_{\mathrm{up}}(t)$ consists of the following: interference from other users in the cell which called intracell interference, the receiver noise $n(t)$, and the received signal from the desired user.

From Figure $2, R_{\text {up }}(t)$ is given by

$$
R_{\mathrm{up}}(t)=\sum_{i=0}^{N-1} a_{i} S_{i}\left(t-\tau_{i}\right)+n(t)
$$

where $a_{i}$ represents the path loss of the $i$ th user, $\tau_{i}$ is the random delay of the $i$ th user signal at the receiver, and $n(t)$ is the additive white Gaussian noise (AWGN) of the receiver.

The signal at the output of the matched filter is given by

$$
Z\left(T_{b}\right)=\frac{1}{T_{b}} \int_{\tau_{0}}^{T_{b}+\tau_{0}} R_{\text {up }}(t) 2 \cos \left(w_{1} t+\theta\right) c_{i}\left(t-\tau_{i}\right) d t,
$$

where $\theta$ is the carrier phase angle in the receiver as shown in Figure 2.

The intracell interference at the output of the matched filter is given by

$$
Z_{\text {int }}(t)=\sum_{i=1}^{N-1} Z_{i}\left(T_{b}\right)
$$

\subsection{Perfect Power Control}

To reduce the near-far problem, as well as the interference from other users and hence to increase the capacity of CDMA WLL system, it is important to apply a power control on the reverse link so that the received power from each user at the BS is controlled to be the constant target power, $S$, [5] where

$$
a_{i}^{2} A_{i}=S \quad \text { for } i=0,1, \ldots, N-1 .
$$

The total noise power is the sum of the intracell interference power and the AWGN power of the receiver.

The AWGN power at the output of the matched filter is given by $[5,7]$ as

$$
\eta=N_{o} R_{b}=\left(\frac{N_{o}}{T_{c}}\right) T_{c} R_{b}=\frac{N_{o} W}{G_{p}},
$$

where $R_{b}=1 / T_{b}$ is the bitrate of the message sequence $b i(t)$, $W=1 / T_{c}$ is the chiprate, and $N_{0} W$ is the noise power at the receiver input.

Thus, after despreading, the noise power $\eta$ is the input noise power decreased by the processing gain $G_{p}=T_{b} / T_{c}$. The intracell interference power is given by [5]

$$
I_{\text {int }}=\operatorname{var}\left(Z_{\text {int }}(t)\right)=\frac{1}{G_{p}} \sum_{i=1}^{N-1} a_{i}^{2} A_{i},
$$

where $\operatorname{var}\left(Z_{\text {int }}(t)\right)$ is the variance of intracell interference at the output of the matched filter.

By applying voice activity detection, users transmit only when speech signal is present. We introduce a voice activity variable (VAF) $v_{i}$ which equals 1 with probability of $\mu$, and equals 0 with probability of $1-\mu$. By multiplying (7) by $v_{i}$ and using (5) so,

$$
\frac{I_{\text {int }}}{S}=\frac{1}{G_{p}} \sum_{i=1}^{N-1} \frac{v_{i} a_{i}^{2} A_{i}}{S}=\frac{1}{G_{p}} \sum_{i=1}^{N-1} v_{i},
$$

the intracell interference to signal power ratio given by (8) is reduced by a factor of $G_{p}$ after the process of matched filtering. Now, we define the ratio $E_{b} / I_{o}$, which is the energy per bit to interference density ratio, where $E_{b}=S T_{b}, I_{0}=$ $I / R_{b}=I T_{b}$, and $I$ is the total interference power (sum of $I_{\text {int }}$ and $\eta$ ) so

$$
\frac{E_{b}}{I_{0}}=\frac{S}{I}=\frac{1}{I_{\text {int }} / S+\eta / S} .
$$

In (8), the summation of $v_{i}$ over $(N-1)$ users may be expressed as

$$
\sum_{i=1}^{N-1} v_{i}=\mu(N-1)
$$

so that

$$
\frac{E_{b}}{I_{0}}=\frac{1}{\mu(N-1) / G_{p}+\eta / S} .
$$

The bit error rate (BER) for the binary phase shift keying (BPSK) modulation can be expressed as

$$
P_{b}=\frac{1}{2} \operatorname{erfc}\left(\sqrt{\frac{E_{b}}{I_{0}}}\right)
$$

where $\operatorname{erfc}(\sigma)$ is the complementary error function [5]. For a required BER, a required $E_{b} / I_{o},\left(E_{b} / I_{o}\right)_{\text {req }}$ can be determined from (12). Given $\left(E_{b} / I_{o}\right)_{\text {req }}$, the maximum number of active users, other than the $i$ th user, that can be supported by the system is given by (11) as

$$
m=\sum_{i=1}^{N-1} v_{i}=\left\lfloor\frac{G_{p}}{\left(E_{b} / I_{0}\right)_{\mathrm{req}}}-\frac{G_{p}}{S / \eta}\right\rfloor,
$$

where $\lfloor x\rfloor$ represent the largest integer that is smaller than or equal $x$. Provided that the number of active users does 


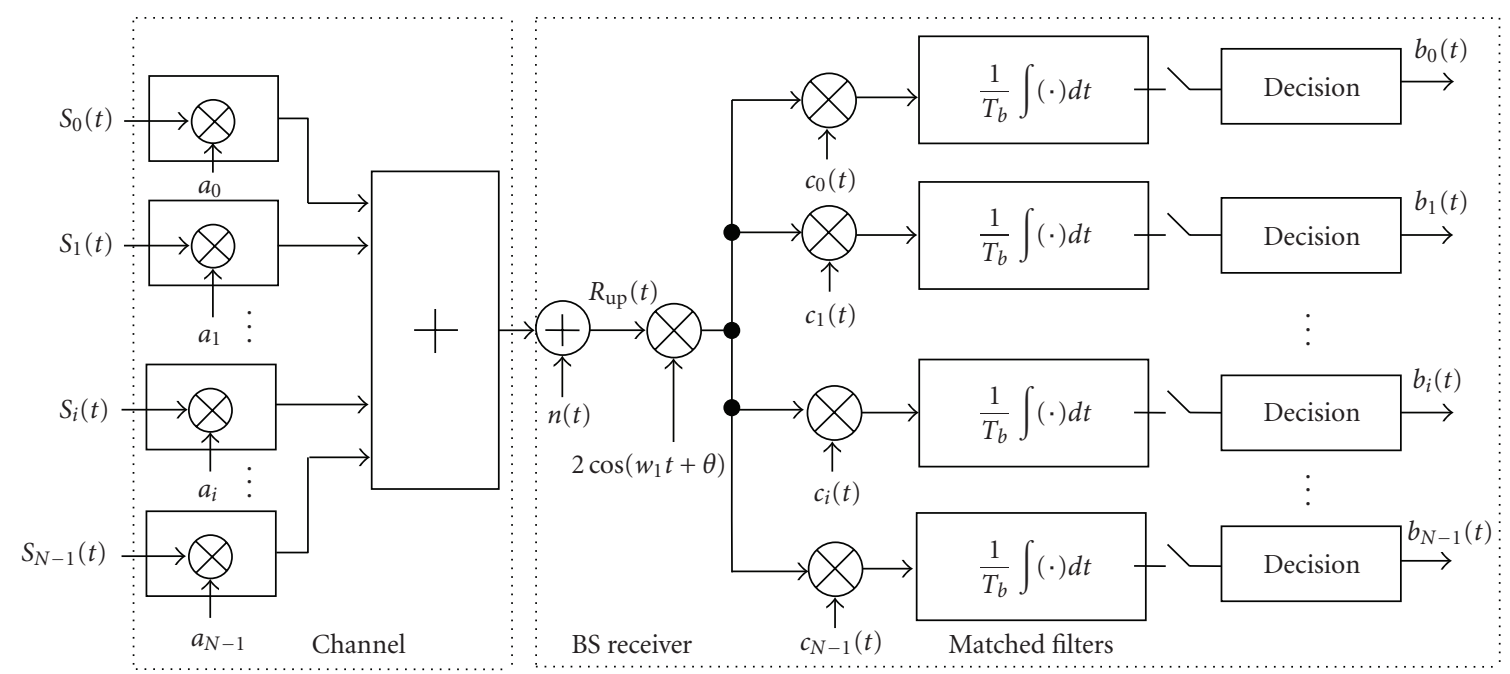

FIGURE 2: Channel and BS receiver block diagram.

not exceed $m$. However, when the number of active users is larger than $m$, the BER will be greater than the required BER, and this situation is referred to as system outage. The outage probability of the single cell system is defined as

$$
P_{\text {out }}=\operatorname{Pr}\left(\text { BER }>\mathrm{BER}_{\text {req }}\right)=\operatorname{Pr}\left(\frac{E_{b}}{I_{0}}<\left(\frac{E_{b}}{I_{0}}\right)_{\text {req }}\right) .
$$

The outage probability is defined as the probability that the number of active users being greater than $m$, that is,

$$
P_{\text {out }}=\operatorname{Pr}\left(\sum_{i=1}^{N-1} v_{i}>m\right)=\sum_{i=1}^{N-1}\left(\begin{array}{c}
N-1 \\
i
\end{array}\right) \mu^{i}(1-\mu)^{N-1-i} .
$$

\subsection{Imperfect Power Control}

In a practical system, the power control is not perfect. So, the received signal power from the $i$ th user at its BS will differ from the target power level $S$ by $\delta_{i} \mathrm{~dB}$. This power error $\delta_{i}$ is a random variable with a standard deviation $\sigma_{e}$. There are several reasons for $\delta_{i}$ being nonzero, such as the power measurement error at the BS and the inability to adjust the subscriber unit transmitted power sufficiently fast to force $\delta_{i}$ to zero $[5,8]$. The signal power at the output of the matched filter for the $i$ th user can be expressed as

$$
S^{\prime}=10^{\delta_{0} / 10} \cdot S,
$$

and the intracell interference power is

$$
I_{\mathrm{int}}^{\prime}=\frac{1}{G_{p}} \sum_{i=1}^{N-1} v_{i} 10^{\delta_{i} / 10} \cdot S .
$$

The intracell interference to signal power ratio at the output of matched filter becomes

$$
\frac{I_{\text {int }}^{\prime}}{S^{\prime}}=\frac{1}{G_{p}} \sum_{i=1}^{N-1} v_{i} 10^{(\delta i-\delta 0) / 10},
$$

where $\delta_{0}$ and $\delta_{i}$ are two mutually independent random variables of power control errors of the signal and the intracell interferers, respectively. By setting $\varepsilon=\delta_{i}-\delta_{0}(18)$ becomes

$$
\frac{I_{\text {int }}^{\prime}}{S^{\prime}}=\frac{1}{G_{p}} \sum_{i=1}^{N-1} v_{i} 10^{\varepsilon / 10}=\frac{I_{\text {int }}}{S} 10^{\varepsilon / 10},
$$

where $\varepsilon$ is a random variable with zero mean and a standard deviation $\sigma_{\varepsilon}=\sqrt{2} \sigma_{e}$. Following the same procedure as in the perfect power control case, the ratio $E_{b} / I_{o}$ can be written as

$$
\left(\frac{E_{b}}{I_{0}}\right)_{\mathrm{imp}}=\frac{1}{I_{\mathrm{int}}^{\prime} / S^{\prime}+\eta / S^{\prime}}=\frac{1}{\left(I_{\mathrm{int}} / S\right) 10^{\varepsilon / 10}+\eta / S^{\prime}} .
$$

In order to evaluate the system performance, we introduce the outage probability that is defined as the probability of a system's BER being greater than $10^{-3}$, that is,

$$
\begin{aligned}
P_{\text {out }} & =\operatorname{Pr}\left(\mathrm{BER}>10^{-3}\right) \\
& =\operatorname{Pr}\left(\left(\frac{E_{b}}{I_{0}}\right)_{\text {imp }}=\frac{S^{\prime}}{I_{\text {int }}^{\prime}+\eta}<\gamma_{\text {req }}\right) \\
& =\operatorname{Pr}\left(\frac{I_{\text {int }}^{\prime}+\eta}{S^{\prime}}>\frac{1}{\gamma_{\text {req }}}\right) \\
& =\operatorname{Pr}\left\{10^{\varepsilon / 10} \sum_{i=0}^{N-1} v_{i}>G_{p}\left(\frac{1}{\gamma_{\text {req }}}-\frac{\eta}{S^{\prime}}\right)\right\},
\end{aligned}
$$

where $\gamma_{\text {req }}$ is the required $E_{b} / I_{o}$ to ensure that the BER is less than $10^{-3}$. If the number of active users inside the cell is $k$, then (22) can be rewritten as

$$
\begin{aligned}
P_{\text {out }}= & \operatorname{Pr}\left\{\left(k 10^{\varepsilon / 10}>G_{p}\left(\frac{1}{\gamma_{\text {req }}}-\frac{\eta}{S^{\prime}}\right)\right) \mid\left(\sum_{i=0}^{N-1} v_{i}=k\right)\right\} \\
& \times \operatorname{Pr}\left(\sum_{i=0}^{N-1} v_{i}=k\right)=P_{1} P_{2} .
\end{aligned}
$$


The probability $P_{1}$ in (23) is given by [5]

$$
P_{1}=Q\left(\frac{G_{p}\left(1 / \gamma_{\mathrm{req}}-\eta / S^{\prime}\right)-k E\left(10^{\varepsilon / 10}\right)}{\sqrt{k \operatorname{var}\left(10^{\varepsilon / 10}\right)}}\right),
$$

where

$$
Q(x)=\frac{1}{\sqrt{2 \pi}} \int_{x}^{\infty} e^{\left(-y^{2} / 2\right)} d y .
$$

The mean of the term $10^{\varepsilon / 10}$ in (24) can be derived as following:

$$
\begin{aligned}
E\left(10^{\varepsilon / 10}\right) & =\int_{-\infty}^{\infty} \exp \left[\frac{\varepsilon \ln (10)}{10}\right] \frac{\exp \left(-\varepsilon^{2} / 4 \sigma_{e}^{2}\right)}{\sqrt{4 \pi \sigma_{e}^{2}}} d \varepsilon \\
& =\exp \left(\sigma_{e} \frac{\ln (10)}{10}\right)^{2},
\end{aligned}
$$

and the variance of the $10^{\varepsilon / 10}$ is given by

$$
\begin{aligned}
\operatorname{var}\left(10^{\varepsilon / 10}\right) & =E\left(10^{\varepsilon / 10}\right)^{2}-\left\{E\left(10^{\varepsilon / 10}\right)\right\}^{2} \\
& =\exp \left(\sigma_{e} \frac{\ln (10)}{5}\right)^{2}-\left(\exp \left(\sigma_{e} \frac{\ln (10)}{10}\right)^{2}\right)^{2}
\end{aligned}
$$

Now, consider the probability that there are $k$ active intracell users, $P_{2}$, which is given by [5]

$$
P_{2}=\operatorname{Pr}\left(\sum_{i=0}^{N-1} v_{i}=k\right)=\sum_{k=0}^{N-1}\left(\begin{array}{c}
N-1 \\
k
\end{array}\right) \mu^{k}(1-\mu)^{N-1-k} .
$$

The performance of the reverse link in a single cell CDMA WLL system, shown in Figure 3, is evaluated for $20 \mathrm{~dB} / \mathrm{dec}$, where WLL has a fixed-to-fixed link so propagation exponent of 2 is used [2], $E_{b} / I_{o}=5 \mathrm{~dB}$ [9], $W=1.25 \mathrm{MHz}, R_{b}=$ $8 \mathrm{Kbps}, \mathrm{VAF}=3 / 8$, a signal-to-AWGN ratio of $20 \mathrm{~dB}$ at the output of the matched filter and a BER outage threshold of $10^{-3}$ were used in the calculations.

For perfect power control and an outage probability of $2 \%$, the single cell system can support up to 89 users/cell as shown in Figure 3. The outage probability of the imperfect power-controlled system having different standard deviations of power control error is also shown in the figure. For an outage probability of $2 \%$ and a standard deviation of power control errors of $2 \mathrm{~dB}$, the system can support 66 users per cell. The capacity degradation, due to imperfect power control, is about $25 \%$.

Table 1 shows the number of users per cell for different values of the standard deviation (STD) of power control errors and the percentage decrease in users due to imperfect power control, for an outage of $2 \%$.

\section{Effect of Imperfect Power Control in Multiple Cell CDMA WLL Systems}

In addition to the intracell interference, there is now interference from neighboring cells, which called intercell

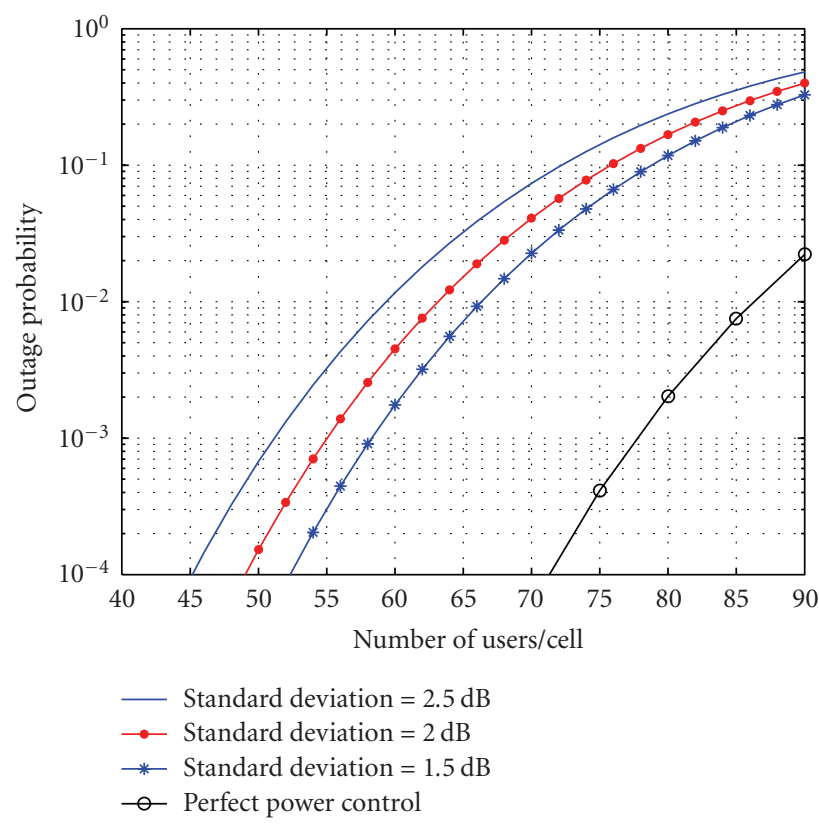

FIgURE 3: Outage probability of a single cell CDMA WLL system.

TABLe 1: Number of users per cell for different values of $\sigma_{e}$, and $P_{\text {out }}=2 \%$.

\begin{tabular}{lcc}
\hline STD & Users/cell & $\%$ decrease \\
\hline $0 \mathrm{~dB}$ & 89 & $0 \%$ \\
$1.5 \mathrm{~dB}$ & 69 & $22.4 \%$ \\
$2 \mathrm{~dB}$ & 66 & $25.8 \%$ \\
$2.5 \mathrm{~dB}$ & 62 & $30.3 \%$ \\
\hline
\end{tabular}

interference. The received signal at the BS includes: the desired signal, intracell interference, the AWGN at the receiver input, and intercell interference. Figure 4 shows the reverse-link communication system, where the arrangement for the transmitter and BS receiver is the same as those shown in Figures 1 and 2, respectively.

The received signal at the BS is given by [5]

$$
R_{\mathrm{up}}(t)=\sum_{i=0}^{N-1} a_{i} S_{i}\left(t-\tau_{i}\right)+\sum_{j=1}^{J-1} \sum_{i=0}^{N-1} a_{i j} S_{i j}\left(t-\tau_{i j}\right)+n(t),
$$

where the intercell interference from the $J-1$ surrounding cells is

$$
\sum_{j=1}^{J-1} \sum_{i=0}^{N-1} a_{i j} S_{i j}\left(t-\tau_{i j}\right),
$$

where $a_{i j}$ represents the effects of path loss, $\tau_{i j}$ is the random time delay of the $i$ th user in the $j$ th cell, and $s_{i j}(t)$ is the signal transmitted by the $i$ th user in the $j$ th cell.

For a particular user, say the zeroth one, the signal at the output of the matched filter is given by

$$
Z\left(T_{b}\right)=a_{0} \sqrt{2 A_{0}} b_{0} \cos \varphi_{0}+Z_{\text {int }}\left(T_{b}\right)+Z_{\text {ext }}\left(T_{b}\right)+Z_{n}\left(T_{b}\right),
$$




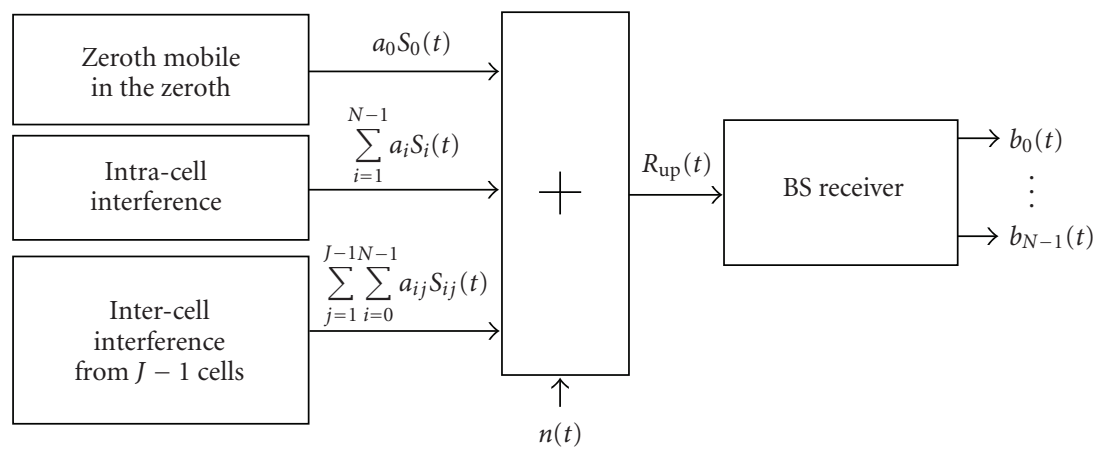

FIGURE 4: Block diagram of the multicell CDMA reverse-link system.

where $\varphi_{0}$ is the carrier phase difference. The first term is the desired signal, the second term is the intracell interference component, the third term is the intercell interference component, while the last term is the AWGN component. The intercell interference at the output of the matched filter is given by

$$
Z_{\mathrm{ext}}(t)=\frac{1}{T_{b}} \int_{0}^{T_{b}} \sum_{j=1}^{J-1} \sum_{i=0}^{N-1} a_{i j} \sqrt{2 A_{i j}} b_{i j}\left(t-\tau_{i j}\right) c_{i j}\left(t-\tau_{i j}\right) .
$$

\subsection{Perfect Power Control}

Similar to the approach in deriving the intracell interference power, the intercell interference power at the output of the matched filter, $I_{\text {ext }}$, can be shown to be

$$
I_{\mathrm{ext}}=E\left(\left(Z_{\mathrm{ext}}\left(T_{b}\right)\right)^{2}\right)=\frac{1}{G_{p}} \sum_{j=1}^{J-1} \sum_{i=0}^{N-1} a_{i j}^{2} A_{i j} .
$$

The intercell interference to signal ratio is given by

$$
\frac{I_{\text {ext }}}{S}=\frac{1}{G_{p}} \sum_{j=1}^{J-1} \sum_{i=0}^{N-1} v_{i j} \frac{a_{i j}^{2} A_{i j}}{S}=\frac{1}{G_{p}} \sum_{j=1}^{J-1} \frac{I_{j}}{S},
$$

where $I_{j} / S$ is the interference to signal power ratio from the $j$ th cell.

Let us consider one of the interfering cells, say cell ${ }_{j}$, where its $\mathrm{BS}$ is at a distance $d$ from $\mathrm{BS}_{0}$ as shown in Figure 5. The interference term $I_{j}$ in (34) is the interference power from all the users in cell $j$ to the $\mathrm{BS}_{0},[5,10]$.

If the interfering user in cell ${ }_{j}$ is located at a distance $r$ from its $\mathrm{BS}$ and $r_{o}$ from the $\mathrm{BS}_{0}$, the interfering user, when active, produces an interference to the $\mathrm{BS}_{0}$ given by [11]

$$
\frac{I\left(r_{o}, r\right)}{S}=\left(\frac{10^{\left(\zeta_{0} / 10\right)}}{r_{0}^{\alpha}}\right)\left(\frac{r^{\alpha}}{10^{\left(\zeta_{m} / 10\right)}}\right)=\left(\frac{r}{r_{0}}\right)^{\alpha} 10^{\zeta / 10} \leq 1,
$$

where the first term is, due to the attenuation, caused by distance and blockage to the given BS, while the second term is the effect of power control to compensate for the corresponding attenuation to the BS of the out-of-cell

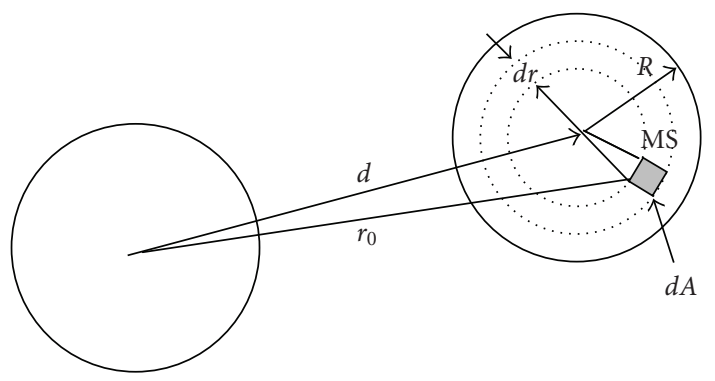

FIGURE 5: CDMA interference calculation.

interferer, $\zeta, \zeta_{0}$, and $\zeta_{m}$ all are random variables with zero mean and standard deviation $\sigma,\left(\zeta=\zeta_{0}-\zeta_{m}\right)$, and $r_{0}$ is given by

$$
r_{0}=\sqrt{d^{2}+r^{2}+2 d r \cos \theta} .
$$

Replace the summation in (34) by an integration over the area of the cell $l_{j}$, so $I_{j} / S$ will be

$$
\frac{I_{j}}{S}=\int_{0}^{2 \pi} \int_{0}^{R} v_{i j} \frac{I\left(r_{0}, r\right)}{S} \cdot \varphi\left(\xi, \frac{r}{r_{0}}\right) \rho d a
$$

where $\rho$ is the user density, assuming $N$ users are uniformly distributed in a circular cell of radius $R, \rho=N / \pi R^{2}, d a=$ $r d r d \theta$ is the unit area in Figure 5, $\Phi\left(\zeta_{0}-\zeta_{m}, r / r_{0}\right)$ is the constraint function for the interfering users in the cell $_{j}$, which can be defined as $[5,11]$

$$
\varphi\left(\xi, \frac{r}{r_{0}}\right)= \begin{cases}1, & \text { if }\left(\frac{r}{r_{0}}\right)^{\alpha} 10^{\xi / 10} \leq 1, \\ 0, & \text { otherwise. }\end{cases}
$$

It is necessary to calculate the mean and variance of the intercell interference power in order to calculate the outage probability. From (35) into (37) and by taking the mean, we obtain [11]

$$
E\left(\frac{I_{j}}{S}\right)=\rho \mu \int_{0}^{2 \pi} \int_{0}^{R}\left(\frac{r}{r_{0}}\right)^{\alpha} E\left(10^{\xi / 10} \cdot \varphi\left(\xi, \frac{r}{r_{0}}\right)\right) r d r d \theta
$$


where $E\left(v_{i j}\right)=\mu$. The variance of $I_{j} / S$ can be given by [11]

$$
\begin{aligned}
& \operatorname{var}\left(\frac{I_{j}}{S}\right) \\
& \quad=\int_{0}^{2 \pi} \int_{0}^{R} E\left[v_{i j}\left(\frac{r}{r_{0}}\right)^{\alpha} 10^{\xi / 10} \cdot \varphi\left(\xi, \frac{r}{r_{0}}\right)\right]^{2} \\
& \quad-\left\{E\left[v_{i j}\left(\frac{r}{r_{0}}\right)^{\alpha} 10^{\xi / 10} \cdot \varphi\left(\xi, \frac{r}{r_{0}}\right)\right]\right\}^{2} \rho r d r d \theta .
\end{aligned}
$$

The total interference-power-to-signal-power ratio for all the surrounding cells, $I_{\text {ext }} / S$, in (34) has a mean and variance of the following:

$$
\begin{gathered}
E\left[\frac{I_{\mathrm{ext}}}{S}\right]=\frac{1}{G_{p}} \sum_{j=1}^{J-1} E\left[\frac{I_{j}}{S}\right], \\
\operatorname{var}\left[\frac{I_{\mathrm{ext}}}{S}\right]=\left(\frac{1}{G_{p}}\right)^{2} \sum_{j=1}^{J-1} \operatorname{var}\left[\frac{I_{j}}{S}\right] .
\end{gathered}
$$

The ratio $E_{b} / I_{o}$ at the output of the matched filter can be written as

$$
\frac{E_{b}}{I_{0}}=\frac{1}{I_{\text {int }} / S+I_{\text {ext }} / S+\eta / S} .
$$

The system performance in terms of the outage probability that has a BER greater than $10^{-3}$ is

$$
\begin{aligned}
P_{\text {out }}=\sum_{k=0}^{N-1}\left(\begin{array}{c}
N-1 \\
k
\end{array}\right) \mu^{k}(1-\mu)^{N-1-k} \\
\times Q\left(\frac{1 / \gamma_{\text {req }}-\eta / S-k / G_{p}-E\left(I_{\text {ext }} / S\right)}{\sqrt{\operatorname{var}\left(I_{\text {ext }} / S\right)}}\right) .
\end{aligned}
$$

\subsection{Imperfect Power Control}

The received signal power $S^{\prime}$ from a user at its BS will differ from the target power level $S$ by $\delta_{0} \mathrm{~dB}$. This error power is a random variable with standard deviation $\sigma_{e}$. Using (16) and (35), the interfering power to received signal power ratio will be

$$
\frac{I^{\prime}\left(r_{o}, r\right)}{S^{\prime}}=\left(\frac{r}{r_{0}}\right)^{\alpha} 10^{\zeta / 10} 10^{\delta i j-\delta_{0}}=\left(\frac{r}{r_{0}}\right)^{\alpha} 10^{\zeta / 10} 10^{\varepsilon / 10},
$$

where $\delta_{i j}$ is the power error for the $i$ th user in cell $j$. The total intercell interference-to-signal ratio is

$$
\frac{I_{\text {ext }}^{\prime}}{S^{\prime}}=\frac{I_{\text {ext }}}{S} 10^{\varepsilon / 10} \text {. }
$$

The ratio $E_{b} / I_{o}$ can be written as

$$
\begin{aligned}
\left(\frac{E_{b}}{I_{0}}\right)_{\text {imp }}= & \frac{1}{I_{\text {int }}^{\prime} / S^{\prime}+I_{\text {ext }}^{\prime} / S^{\prime}+\eta / S^{\prime}} \\
& +\frac{1}{\left(I_{\text {int }} / S\right) 10^{\varepsilon / 10}+\left(I_{\text {ext }} / S\right) 10^{\varepsilon / 10}+\eta / S^{\prime}} .
\end{aligned}
$$

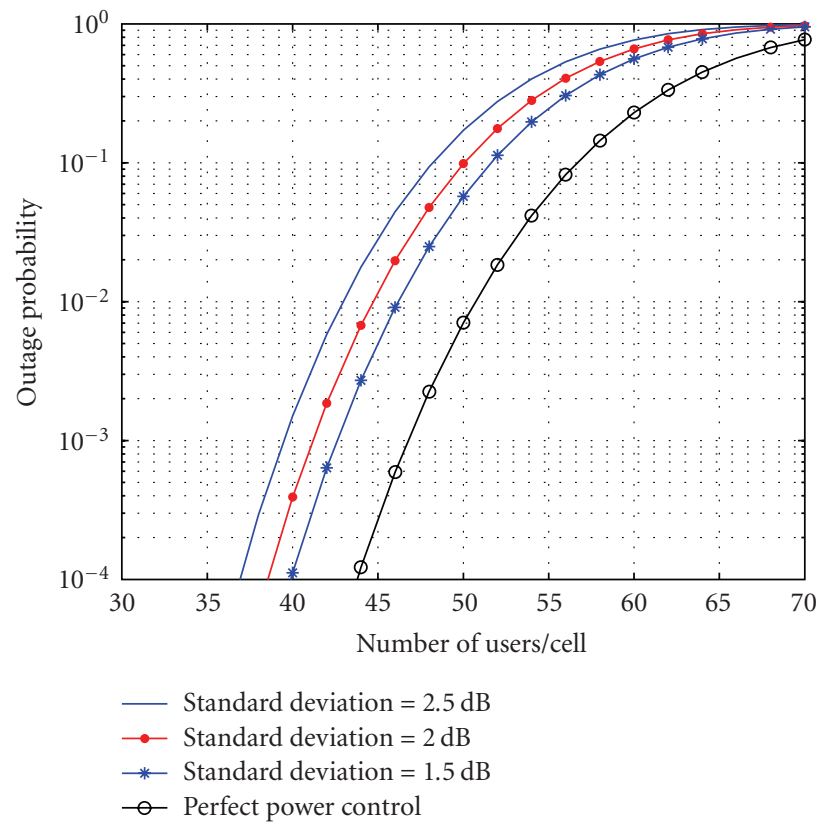

FIgUre 6: Outage probability of the multiple cell CDMA WLL system.

Following the same procedure as employed in the perfect power control case, $P_{\text {out }}$ is found as

$$
\begin{aligned}
P_{\mathrm{out}}=\sum_{k=0}^{N-1}\left(\begin{array}{c}
N-1 \\
k
\end{array}\right) \mu^{k}(1-\mu)^{N-1-k} \\
\quad \times Q\left(\frac{1 / \gamma_{\mathrm{req}}-\eta / S^{\prime}-k / G_{p}-E\left(I_{\mathrm{int}}^{\prime} / S^{\prime}\right)-E\left(I_{\mathrm{ext}}^{\prime} / S^{\prime}\right)}{\sqrt{\operatorname{var}\left(I_{\mathrm{int}}^{\prime} / S^{\prime}\right)+\operatorname{var}\left(I_{\mathrm{ext}}^{\prime} / S^{\prime}\right)}}\right),
\end{aligned}
$$

where $I_{\text {int }}^{\prime} / S^{\prime}$ and $I_{\text {ext }}^{\prime} / S^{\prime}$ are two independent Gaussian distributed random variables, whose mean and variance may be expressed as

$$
\begin{gathered}
E\left[\frac{I_{\text {int }}^{\prime}}{S^{\prime}}+\frac{I_{\text {ext }}^{\prime}}{S^{\prime}}\right]=E\left[\frac{I_{\text {int }}}{S} 10^{\varepsilon / 10}\right]+E\left[\frac{I_{\text {ext }}}{S} 10^{\varepsilon / 10}\right], \\
\operatorname{var}\left[\frac{I_{\text {int }}^{\prime}}{S^{\prime}}+\frac{I_{\text {ext }}^{\prime}}{S^{\prime}}\right]=\operatorname{var}\left[\frac{I_{\text {int }}}{S} 10^{\varepsilon / 10}\right]+\operatorname{var}\left[\frac{I_{\text {ext }}}{S} 10^{\varepsilon / 10}\right] .
\end{gathered}
$$

The performance of the reverse-link CDMA WLL system is shown in Figure 6. For an outage probability of $2 \%$, the perfect power-controlled system can support 52 users/cell for a VAF of $3 / 8$. The number of users per cell for different values of the standard deviation of power control errors and the percentage decrease in users, due to imperfect power control, are displayed in Table 2 for an outage of $2 \%$.

\section{Effect of Imperfect Sectorization in CDMA WLL Systems}

In the case of perfect directional antennas, there is a sharp separation between the sectors. Due to overlap and sidelobe 
TABLe 2: Number of users per cell for different values of $\sigma_{e}$, and $P_{\text {out }}=2 \%$.

\begin{tabular}{lcc}
\hline STD & Users/cell & $\%$ decrease \\
\hline $0 \mathrm{~dB}$ & 52 & $0 \%$ \\
$1.5 \mathrm{~dB}$ & 47 & $9.6 \%$ \\
$2 \mathrm{~dB}$ & 46 & $11.5 \%$ \\
$2.5 \mathrm{~dB}$ & 44 & $15.4 \%$ \\
\hline
\end{tabular}

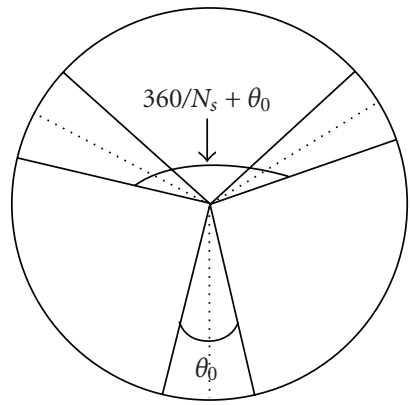

(a)

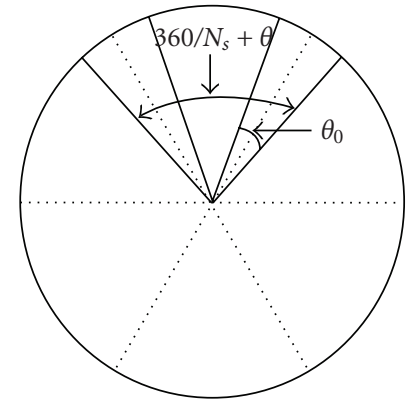

(b)
FIGURE 7: Imperfect sectorization: (a) three sector; (b) six sector per cell.

of practical antenna, the BS still receives some interference from users in other sectors $[11,12]$.

Figure 7 shows a sectorized cell arrangement having $N_{s}$ overlapping sectors, each with an angle of $\left(2 \pi / N_{s}\right)+\theta_{0}$, where $\theta_{0}$ is the overlapping angle. If there is no overlapping, that is, $\theta_{0}=0$, then $1 / N_{s}$ of the total interference is received, and the capacity gain, due to sectorization, is $N_{s}$ times that of an unsectorized cell.

In a sectorized cell, only $\left(2 \pi / N_{s}\right)+\theta_{0} / 2 \pi$ of the total interference from the surrounding is received. For this condition, the capacity gain, due to sectorization, is $2 \pi /\left(2 \pi / N_{s}\right)+\theta_{0}$ times that of the unsectorized cell.

We define the efficiency of sectorization $e_{s}$ as the ratio of the capacity gain with the sector antennae pattern having an overlapping angle $\theta_{0}$ to the nonoverlapping antennae pattern of $2 \pi / N_{s}$, so

$$
e_{s}=\frac{2 \pi /\left(2 \pi / N_{s}+\theta_{0}\right)}{2 \pi /\left(2 \pi / N_{s}\right)}=\frac{2 \pi / N_{s}}{2 \pi / N_{s}+\theta_{0}} .
$$

So, the imperfect sectorization capacity gain $G_{\text {imp }}$ will be

$$
G_{\text {imp }}=N_{s} e_{s} .
$$

In WLL system, due to fixed-to-fixed link, six sectors per cell arrangement can be used, the capacity gain due to sectorization and its corresponding sectorization efficiency for different values of overlapping angle $\theta_{0}$ in degrees is shown in Figure 8. From the figure, the interference on the reverse link increases as the overlapping angle $\theta_{0}$ is increased, which causes the capacity gain and sectorization efficiency to decrease proportionally. Increasing $\theta_{0}$ from $0^{\circ}$ to $5^{\circ}$ causes the capacity gain to decrease from 6 to 5.53, while the corresponding sectorization efficiency drops from $100 \%$ to $92.3 \%$.

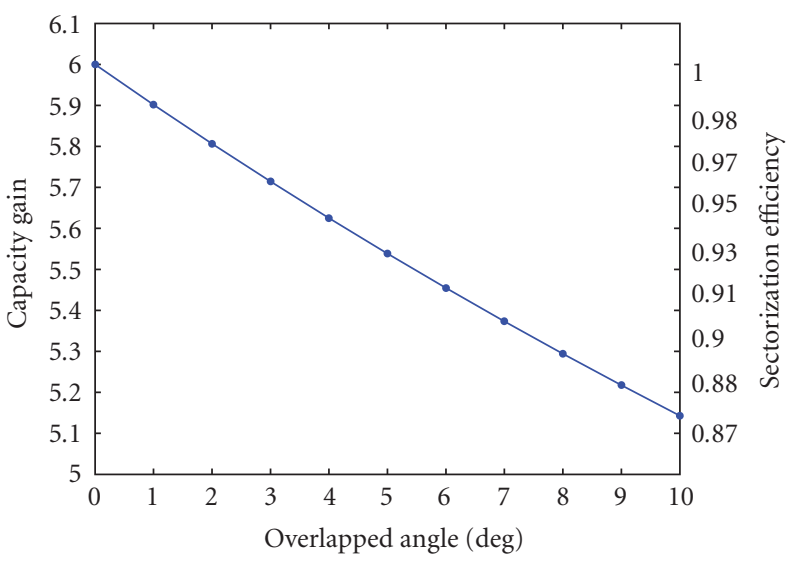

FIGURE 8: Reverse-link sectorization gain and efficiency as a function of the overlapping angle.

\section{Conclusion}

CDMA technology has the potential to provide a significant improvement in the capacity of WLL systems compared with FDMA and TDMA systems. However, this improvement is dependent upon the effectiveness of the power control system, especially on the reverse link. In this paper, a theoretical model to evaluate the reverse-link capacity of CDMA WLL systems in terms of outage probability, taking into account the power control error, is obtained.The results show that the capacity degradation, due to imperfect power control, is about $25.8 \%$, and $11.5 \%$ for single cell and multiple cell systems, respectively. The effect of imperfect sectorization on the performance of CDMA WLL systems is also considered. The interference on the reverse link increases as the overlapping angle is increased, which causes the capacity gain and sectorization efficiency to decrease proportionally as shown in Figure 8.

\section{References}

[1] W. Webb, Introduction to Wireless Local Loop, Artech House, Norwood, Mass, USA, 1998.

[2] D. Lee and C. Xu, "The effect of narrowbeam antenna and multiple tiers on system capacity in CDMA wireless local loop," IEEE Communications Magazine, vol. 35, no. 9, pp. 110114, 1997.

[3] H. Stellakis, A. Giordano, A. Aksu, and W. Biagini, "Reverse link performance of wireless local loop CDMA networks," IEEE Communications Letters, vol. 4, no. 2, pp. 49-51, 2000.

[4] P. Jung, P. W. Baier, and A. Steil, "Advantages of CDMA and spread spectrum techniques over FDMA and TDMA in cellular mobile radio applications," IEEE Transactions on Vehicular Technology, vol. 42, no. 3, pp. 357-364, 1993.

[5] R. Steele, C.-C. Lee, and P. Gould, GSM, cdmaOne and $3 G$ Systems, John Wiley \& Sons, New York, NY, USA, 2001.

[6] W. C. Y. Lee, “Overview of cellular CDMA," IEEE Transactions on Vehicular Technology, vol. 40, no. 2, pp. 291-302, 1991.

[7] C.-C. Lee and R. Steele, "Effect of soft and softer handoffs on CDMA system capacity," IEEE Transactions on Vehicular Technology, vol. 47, no. 3, pp. 830-841, 1998. 
[8] W.-M. Tam and F. C. M. Lau, "Analysis of power control and its imperfections in CDNA cellular systems," IEEE Transactions on Vehicular Technology, vol. 48, no. 5, pp. 1706-1717, 1999.

[9] V. K. Garg and E. L. Sneed, "Digital wireless local loop system," IEEE Communications Magazine, vol. 34, no. 10, pp. 112-115, 1996.

[10] K. I. Kim, “CDMA cellular engineering issues," IEEE Transactions on Vehicular Technology, vol. 42, no. 3, pp. 345-350, 1993.

[11] M. G. Jansen and R. Prasad, "Capacity, throughput, and delay analysis of a cellular DS CDMA system with imperfect power control and imperfect sectorization," IEEE Transactions on Vehicular Technology, vol. 44, no. 1, pp. 67-75, 1995.

[12] A. Ahmad, "A CDMA network architecture using optimized sectoring," IEEE Transactions on Vehicular Technology, vol. 51, no. 3, pp. 404-410, 2002. 

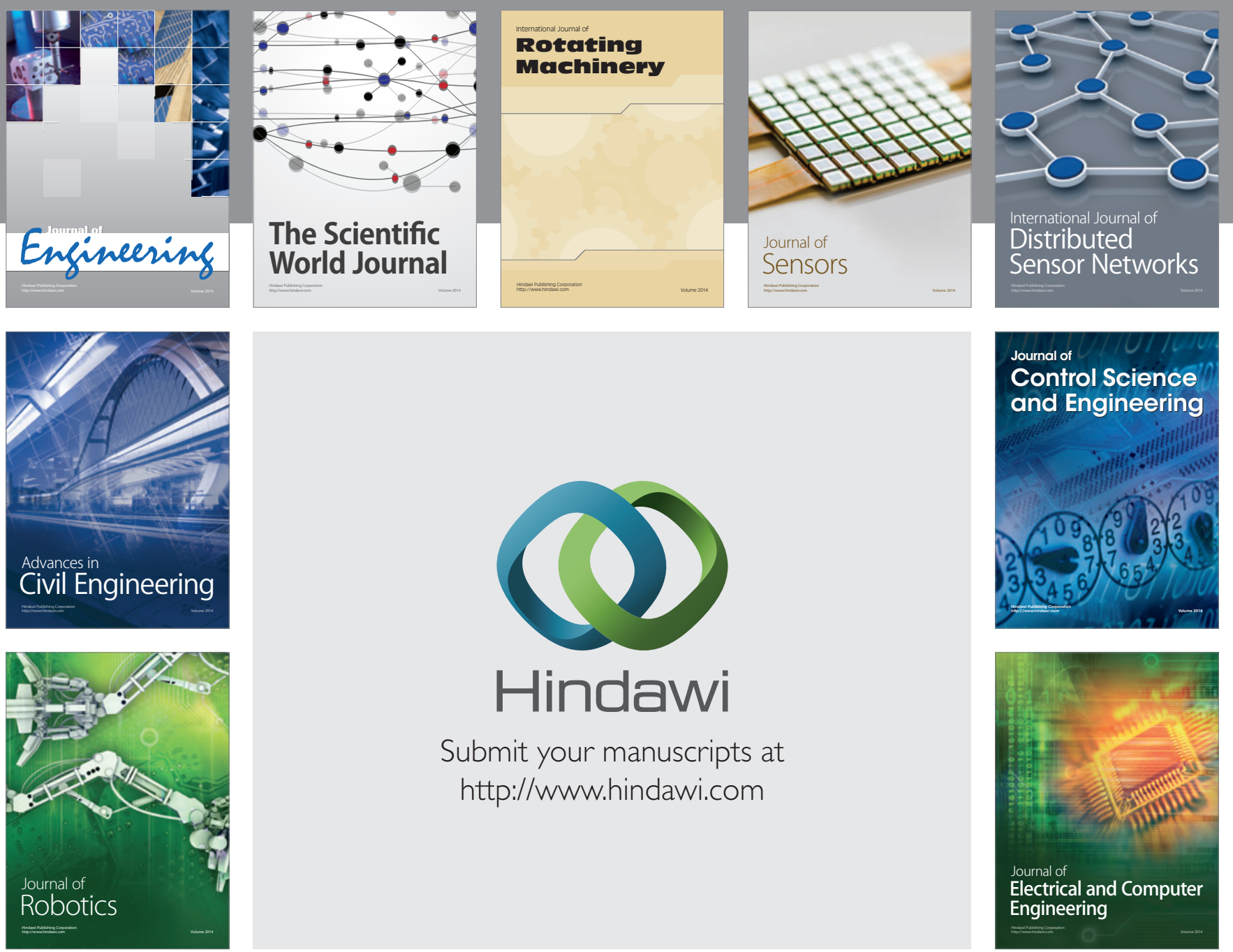

Submit your manuscripts at

http://www.hindawi.com
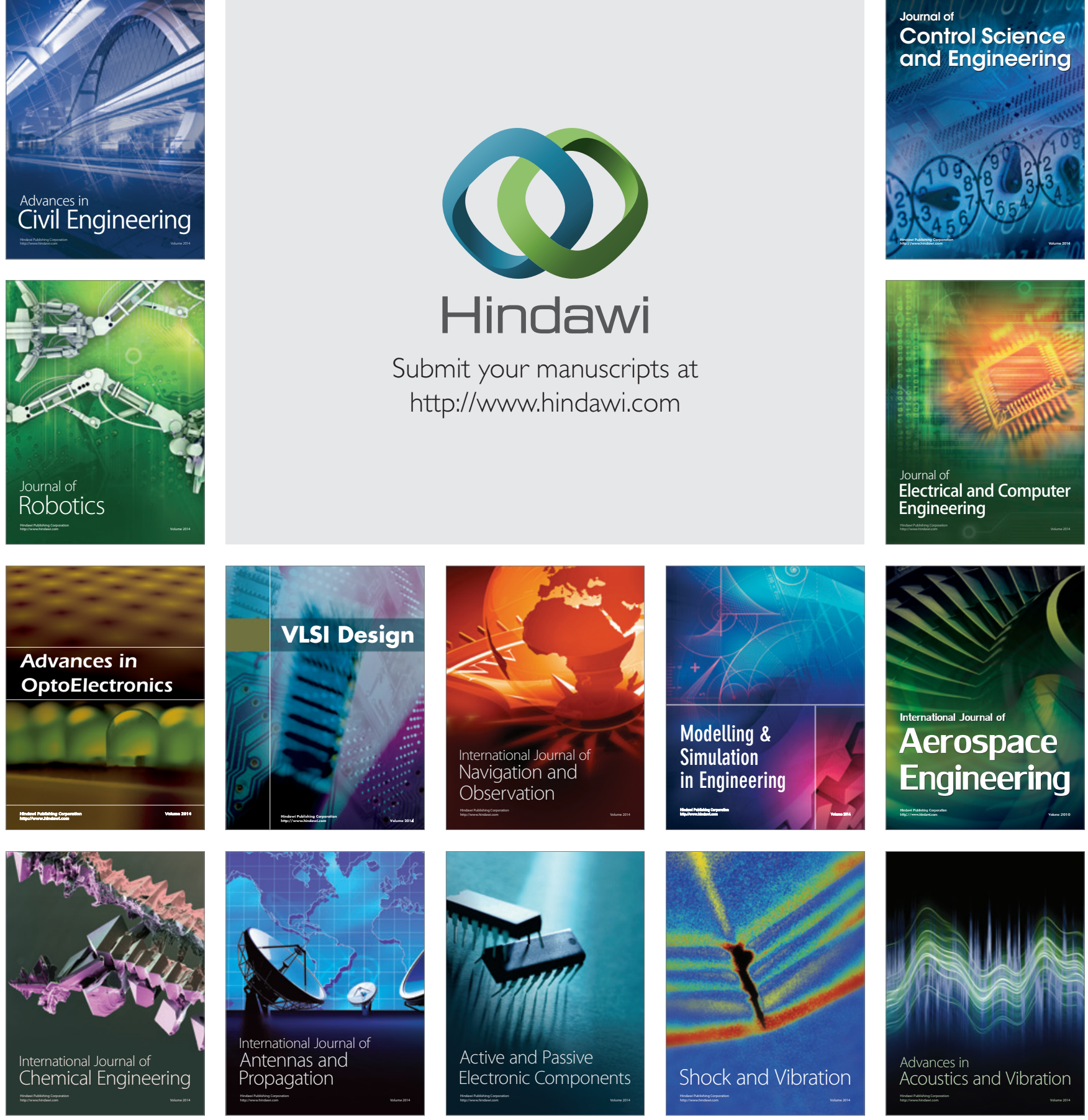